$$
\text { "arkinemet" — 2004/7/23 — 13:48 — page } 1 \text { — }
$$

\title{
Dynamic methods in teaching geometry at different levels
}

\author{
TAMÁS ÁrKI and IstváN KriszTin NÉMET
}

Abstract. In this paper we summarize and illustrate our experiences on DGS-aided teaching geometry of the courses "Computer in mathematics" and "Mathematical software" held for students at Juhász Gyula Teacher Training College of University of Szeged. Furthermore, we show examples from our grammar school experiences too. The figures in this paper were made by using Cinderella ([19]) and Euklides ([21]).

Key words and phrases: computer-aided teaching geometry, Dynamic Geometry Software (DGS).

ZDM Subject Classification: U50, U70, G10, D50, N80, N90.

\section{Introduction}

According to the quick growth of capacity of computers plenty of software appeared which support performing different types of geometrical constructions. These software play an increasing role in various domains and levels of teaching geometry from primary school to higher education. These dynamic tools give the opportunities to perform constructions, to produce sequence of figures fast and precisely and to change the given geometrical construction interactively. The comprehensive name of these systems is Dynamic Geometry Software (DGS) (for example see $[18,19,21,22])$.

Realizing these opportunities we have been using DGS for several years in teaching our students in the courses "Computer in mathematics" and "Mathematical software" at Juhász Gyula Teacher Training College of University of Szeged

Copyright (C) 2004 by University of Debrecen 


$$
\text { "arkinemet" — 2004/7/23 — 13:48 — page } 2 \text { — \#2 }
$$

in order to make them be able to apply dynamic tools in their teaching practice and problem solving. We also had the opportunity to apply DGS-aided teaching geometry at Ságvári Endre Teacher Training Grammar School of University of Szeged.

The present paper is structured into three parts. In the first part we briefly summarize the most important features of DGS. The second part provides a brief account of the most important didactical aspects of DGS, the advantages and problems with their usage in education. A great number of studies (e.g. $[1,5,7,12,13])$ deal with the theoretical background of the application of DGS in the classroom, so the present paper does not undertake to give a detailed analysis. In the final part we concentrate on examples from plane geometry treated in our courses with the aim of illustrating the possible domains and goals of using dynamic methods and also our experiences in the classroom application of DGS. In consideration of the fact that our courses work with small groups, we do not have enough data to make statistical interferences. Nevertheless, we are hopeful that most geometry teachers will find the examples relevant and useful to their work. For application in solid geometry see [3] or [17].

\section{The features of DGS}

In this paper we emphasize only the most important features of DGS from the didactical point of view. One can find a detailed description in [10] or [24].

(1) Interactive constructions. The result of the construction appears immediately in interactive form on the screen. The steps of the construction can be annulled and can be replayed step by step. Thus, one can investigate each phase of the construction.

(2) Derivative structure. Every construction is built on base-points, which can be placed anywhere on the plane. Derivative points are linked to base-points by constructions.

(3) Interactive structure modification. Interactive dragging of the base-points is automatically followed by the modification of the constructions without re-performing them ("dragmode", see details in [1]).

(4) Macro operations. In order to facilitate constructions DGS offers "macro operations" which condense the steps of constructions into one command. See details in [8]. 


$$
\text { "arkinemet" — 2004/7/23 — 13:48 — page } 3 \text { — \#3 }
$$

(5) Locus of points. We run a base-point (or a semi-dragable point) along a given curve (such as line-segment, ray, straight line, circle or other conic) while we visualize the trace of a chosen derivative point. See a detailed analysis in [6].

(6) Animation. While a base-point (or a semi-dragable point) is running along a given curve (such as line-segment, ray, straight line or circle) the result of the actual construction is animated according to the current position of the running point.

\section{Didactical aspects}

Applying DGS in education raises many new opportunities and questions.

(1) Well-structured constructions. In general, DGS hides the auxiliary lines of built-in constructions, which enables to perform clear and embraceable constructions, so the students can pay attention to the substance of the problem considered.

(2) Early application. On the other hand, built-in constructions may cause a superficial knowledge if students do not have enough practice in elementary constructions. This is a notable danger mainly in elementary school and groups of limited abilities. However, DGS might not provide an exclusive environment, so other tools need to be used at the same time $([1])$.

(3) Open problem stating. The usage of DGS calls for a new kind of phrasing of exercises, since the problem-solving is essentially based on computer-aided experiments and phrasing the realized experiences. See our examples or [13].

(4) Abstraction. By the interactivity of DGS, students have the opportunity to think over the problems, and to construct new exercises. See Examples 2 and 5 .

(5) Singular cases. In general, DGS does not handle correctly those constructions which have infinitely many solutions or other extreme "limit" cases (e.g. the circles in touch in Example 3 at software Euklides). A separate discussion is needed in each singular case. On the other hand, in cases with a finite number of solutions the dynamic behavior of the solutions can be wrong at some software products. A simple example for this "dynamic mistake" can be found in [5].

(6) Experiment-conjecture-proof. The result of computer-aided experiments is a mathematical conjecture, which must be proved or disproved (see Example 7). 


$$
\text { "arkinemet" — 2004/7/23 — 13:48 — page } 4 \text { - \#4 }
$$

The imprecision of the visualization also calls attention to the importance of a proof.

(7) Organization of the classroom activity. At grammar school groups it is hard to ensure computer laboratories for each lesson. This problem can be partly resolved by using a projector. In higher education, we work with small groups, so each student can use a computer individually.

(8) The role of the teacher. The classical teaching tasks are extended. The teacher is in charge of structuring the "experiments" (see Examples 9-11) and making the dynamic tools part of the classroom culture. The teacher's work is very complex, so previous preparation entails time inflation (see e.g. $[5,9,13])$.

(9) The students' interactions with DGS. Within the interactive learning environment we have to take into account the students' typical interactions with DGS, such as the non-reflected utilization of tools, the deviation from the given objectives - not to mention the importance of the screen product at the expense of correction the construction $([5,7])$.

\section{Applying DGS in education}

In this paragraph some examples taken from our courses illustrate the application of DGS in various domains of teaching geometry. We hope that the chosen examples demonstrate the facilities of DGS in education from primary school to higher education. Some of the presented topics are examined in details in [23].

\subsection{Elementary discoveries with DGS}

The basic cases of applying DGS are the following:

- experimental discovery of remarkable theorems of pure geometry, like examination of the medians, internal bisectors, incircle, circumcircle, excircles, Euler- and Simson-lines of a triangle and the like. See Examples 1 and 2;

- teaching the connections of geometrical transformations. See Example 4;

- "interactive analysis": discussing the solutions of constructions by dragging the basis points;

- "the pattern of two loci" ([15]): discussing the solutions of constructions by replaying the steps. See Example 3. 


$$
\text { "arkinemet" — 2004/7/23 — 13:48 — page } 5 \text { — \#5 }
$$

ExAmple 1 (An example on tiling). Construct a convex quadrangle and its bimedians. Reflect the quadrangle and the bimedians in the midpoints of the sides. Repeat the construction refer to the image quadrangles. Examine the result by dragging the basis points! Formulate your conjectures about the set of the bimedians! What happens if the original quadrangle becomes a concave one during the dragging?

The students can soon convince themselves that the quadrangles form a tessellation. On the other hand, the lines containing the bimedians form a tessellation of congruent parallelograms (Figure 1). Furthermore, we can call the students' attention to the fact that the parallelogram and the quadrangle are congruent by dissection.

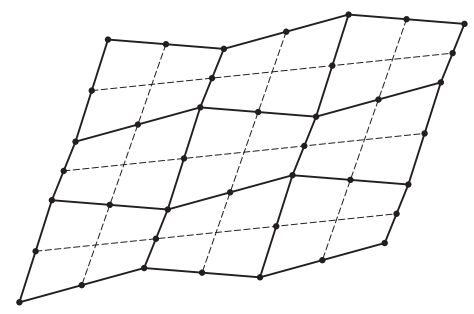

Figure 1. Tiling of congruent quadrangles

EXAMPLE 2 (Interactive study of the Lemoine-point of a triangle). Construct a triangle, its medians and internal bisectors. Reflect the lines in the triangle's corresponding bisectors. Examine the result of the construction by dragging the basis points. Formulate your conjectures! Find triangles for which the conjectures can be proved easily! Try to generalize the problem!

Students can make a conjecture that the reflected images of the rays (the so called "symmedians") are concurrent. Their common point is called the Lemoinepoint of the triangle $([20,25])$.

The interactivity of DGS accords the students every facilities for generalizing the problem. Consider the lines joining an arbitrary point $P$ to the triangle's three vertices ( $P$ differs from the vertices) by dragging $P$. Reflect the lines in the triangle's corresponding bisectors. One can conjecture that the reflected images of the lines are also concurrent (Figure 2) "for the most part of the cases". One can prove by Ceva's theorem $([4,16])$ that if $P$ is not on the circumcircle then the lines in question are concurrent $([20,25])$. 
If $P$ is on the circumcircle, an easy computation of angles shows that the reflected lines are parallel. Due to the simplicity of the proof, the latter case is a good example for finding new problems to solve in grammar school groups by using DGS.

ExAmple 3 (Analysing constructions by DGS). Given a line and two points. "Construct" a parabola through the points whose directrix is the given line! How many parabolas can we obtain this way?

By "construction" we mean the visualization of the parabola by using an apt built-in tool of DGS. In the software Euklides it is sufficient to give the focus and directrix of the parabola. It is easy to construct the focus (Figure 3). We mention two ways to analyse this exercise. The first one is based on interactive modifying of the input data. Students modify the parameters and DGS follows and plots the changes simultaneously. Thus, one can immediately conjecture about the number of solutions with the given input data and way of construction. In particular, this so called "interactive analysis" is effective in primary school groups. Another way is based on replaying the construction. If we study the construction step by step then it is easy to make it clear whether the result of the given step is unique or not. This way of discussion enhances "the pattern of two loci" ([15]), against "interactive analysis" which is based only on visualization.

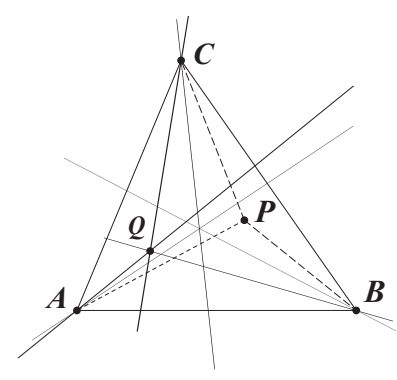

Figure 2

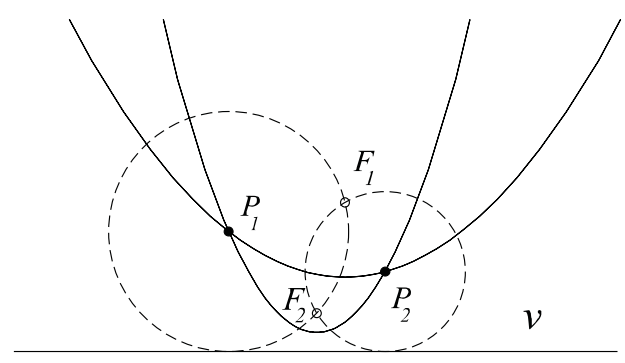

Figure 3

ExAmple 4 (Product of isometries). Construct two intersecting lines $\left(t_{1}, t_{2}\right)$ through a point $O$. Reflect a triangle (or any object) consecutively in $t_{1}$ and $t_{2}$. Which geometrical transformation carries the original triangle to the final triangle? What happens if we modify the position of the lines $t_{1}$ and $t_{2}$ while their angle of inclination is unchanged? Formulate your conjectures and verify them! 


$$
\text { "arkinemet" — 2004/7/23 — 13:48 — page } 7 \text { - \#7 }
$$

Our practical experience is that the students understand easily that the product of these reflections is a rotation. On the other hand, it is harder to understand that if we express a given rotation as the product of two reflections, then one of the axes can be arbitrarily chosen through the centre ([4]), and the other one is determined. In the construction associated with Figure 4 we give the direction of the $t_{1}$ by dragging the point $S$. Thus, the students can observe in motion that the product of the reflections in $t_{1}$ and $t_{2}$ expresses the same rotation in every position of $S$.

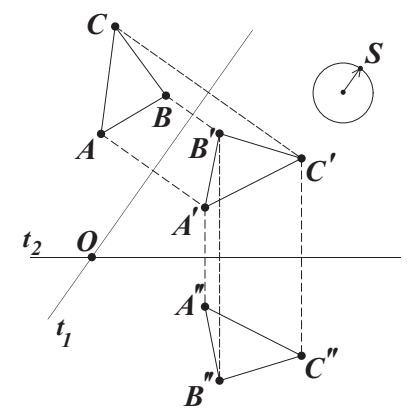

Figure 4. Obtaining of a rotation as a product of two reflections

\subsection{Experiment-conjecture-proof}

Now we illustrate the usefulness of DGS in computer-aided problem-solving. The exercises come from the following domains:

- geometric locus-questions (see Example 5);

- exercises with transformations which are not part of the normal curriculum, such as axial affinity, inversion, polarity (see Example 6);

- exercises for "model-changing" (see Example 8);

- exercises to emphasize the necessity of a proof (see Example 7).

ExAMPlE 5 (Locus with DGS). Given a circle (by its centre $O$ and radius) and an external point. Construct circle which is in contact the given circle and contains the given point. By modifying the tangent-point on the given circle examine the locus of the constructed circle. Formulate your conjectures and try to prove them! Drag the given external point into the given disk and study the changes! What do we find? Explain it! ([23]) 
If a circle containing the point $P$ touches the given circle $k$ at point $F$, then its centre $Q$ is on the line $O F$ and on the perpendicular bisector of the segment $P F$. The locus of $Q$ can be visualized by "running" $F$ on $k$ (see Figure 5.a). Students can make a conjecture that $Q$ traces a hyperbola. Knowing the steps of the construction they can prove easily that the foci of the hyperbola are $O$ and $P$ and the length of its transverse axis is the radius of $k$. It can be interactively visualized that any point on the hyperbola belongs to the locus.

If $P$ is inside the circle, then point $Q$ is on an ellipse (or circle), whose foci are also $O$ and $P$ (see Figure 5.b).

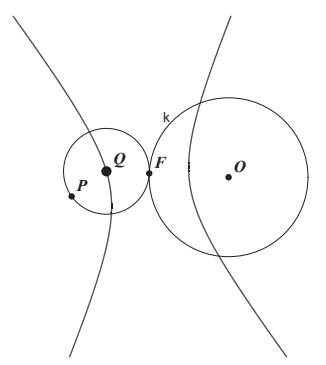

Figure 5.a

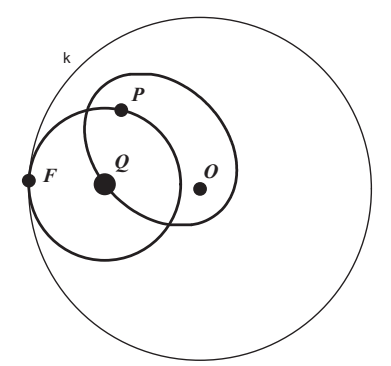

Figure 5.b

Example 6 (Visualizing a conic). Given the foci and a point of an ellipse. Construct a rhombus the sides of which touch the ellipse and one of the sides contains the given point of the ellipse. Taking the trace, draw the ellipse as the affine image of a suitable circle.

A possible solution can be started by constructing the axes of the ellipse. The circle having the major axis as a diameter can be transformed to the ellipse by using a suitable axial affinity. Thus, the tangents of the circle become tangents of the ellipse. If the students performed the construction on paper, they could hardly verify this solution because they could not visualize the ellipse. The application of DGS can help to resolve this problem: the ellipse can be visualized by using "locus of points" (see Figure 6). Hence the trace visualization is an effective method to motivate students in solving problems on conics.

EXAMPLE 7 (The necessity of a proof). Let us consider the circles containing two given points $A$ and $B$. Consider the point(s) on each circle whose distance from the perpendicular bisector of the segment $A B$ is maximal. Find the locus of these point(s)! 


$$
\text { "arkinemet" — 2004/7/23 — 13:48 — page } 9 \text { - \#9 }
$$

While performing the construction, the students are disposed to believe that the locus is the union of those two parabolas whose common directrix is the perpendicular bisector of the segment $A B$, and the axis points are $A$ and $B$, respectively. Figure 7 shows the mentioned parabolas and the locus simultaneously. It is clear that the students' common conjecture is false, which calls their attention to the importance of a proof. By using coordinates it is easy to verify that the locus is a hyperbola.

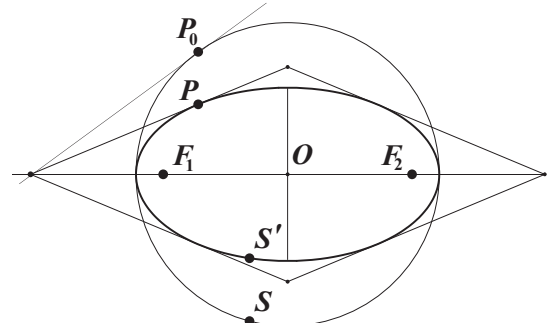

Figure 6

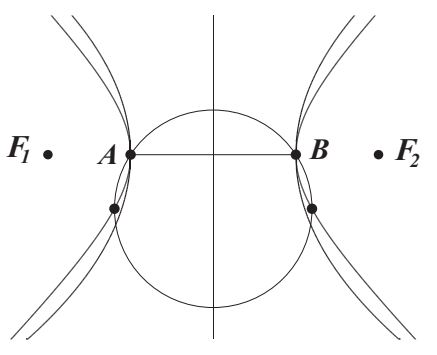

Figure 7

EXAMPLE 8 ("Model-changing"). In the Cartesian coordinate plane, the line $e$ passes through the point $(1,1)$ and the line $f$ passes through the point $(-1,1)$. Given that the difference of the slopes of the two lines is 2, find the locus of the intersection of the lines $e$ and $f$. (Problem B3616 in [11])

This is a simple exercise, but it can be more exciting if we search for the locus by DGS (see Figure 8). To "translate" the exercise into the "language of the DGS" the students have to think over the geometrical meaning of slope and its implementation in the given DGS. This kind of "model-changing" is an effective tool to improve the students' ability for creative problem-solving because the "translation" needs to split the exercise into components and to integrate them with the new model.

\subsection{Concept-formation by DGS}

It is easy to perform animated constructions using DGS. This feature can be applied effectively in forming new concepts. As an illustration we show mathematical structures composed of curves with given properties. The fast and effective graphical representation of these structures were inconceivable before. The typical domains: 
- the concept of envelope (see Examples 9 and 10);

- the concept of coaxal circles (see Example 11).

Example 9 (Envelope of Simson lines). Construct a triangle and project an arbitrary point of its circumcircle to the lines of the sides. What can we say about the position of the pedal points? Search for particular cases in which the conjecture can be proved easily.

As it is known $([4,16])$, the three pedal points lie on a straight line, the so-called Simson line. Now run the given point along the circle. Visualizing the phases of the animation simultaneously, we get a very spectacular figure (Figure 9). The envelope of the Simson lines is a hypocycloid. For details see $[2,20,25]$.

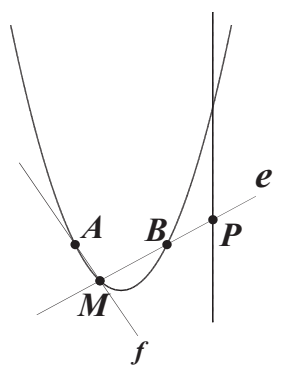

Figure 8

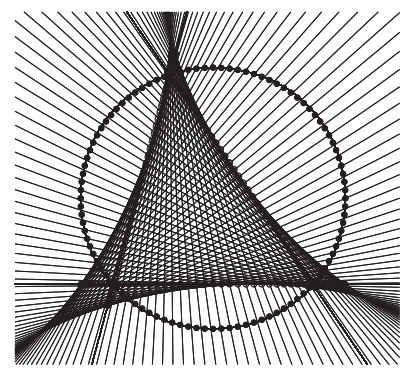

Figure 9

Example 10 (Polarity). Consider the polarity with respect to a given circle. Given another circle and a point on it. Construct the image of this point under the polarity! Visualize the set of the image lines by animation of the point moving on its base circle! Drag the basis points! What is the envelope of the image lines? What relationship can you find between the position of the circle and the envelope?

We can conclude that the envelope is a hyperbola, an ellipse or a parabola according to the centre of the base circle of polarity, whether it is outside, or inside, or on the given circle, respectively ([14]). The latter case can be seen on Figure 10. 


$$
\text { "arkinemet" — 2004/7/23 — 13:48 — page } 11 \text { — \#11 }
$$

ExAmple 11 (Pencil of coaxal circles). Given two intersecting circles and the line containing their common points. Construct a circle whose centre lies on this line and radius is the length of the tangent from this point to one of the given circles! Run the centre along the line! Search for connection between the circles we have got! Formulate your experiences! How can we generate the set of circles with the same property if the two given circles are tangent or nonintersecting?

We visualize the pencil of coaxal circles $([4])$ as the set of circles being orthogonal to both given circles. Figure 11 shows the nonintersecting case.

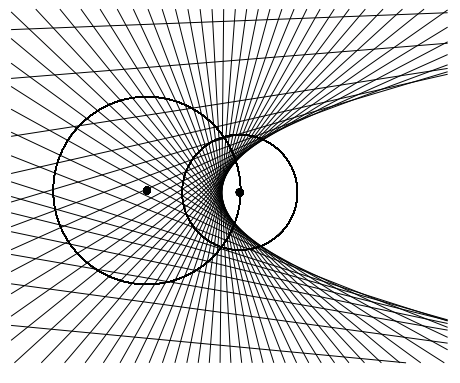

Figure 10

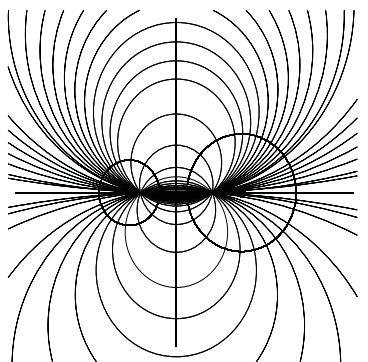

Figure 11

\section{Summary}

Our practical experience is that using DGS provides new possibilities in teaching geometry. The consequences of using the interactive constructive environment are that students get practice in experimental study of geometrical problems and then become able to formulate conjectures ([9]). At the same time, it is the teacher's responsibility that the application of DGS should not be exclusive, should not substitute real tools, but should help to form the precise mathematical thinking. Instead of the closed phrasing of problems (Prove that ...), DGS supports constructive phrasing (Study ..., Search for connection ..., Examine by dragging ..., What relationship can you find between ...) and problem-solving prepared by experiments, thus it highly improves the students' creativity. At this point the role of the teacher appears again, as she (or he) is the one in charge of motivating students to find out why a formulated conjecture is true, and showing the importance of a proof too. To attain the usage of the preferred software and the dynamic approach initially requires plenty of time at the first glance, but we are confident that we get back this time at applications. 


$$
\text { "arkinemet" — 2004/7/23 — 13:48 — page } 12 \text { — \#12 }
$$

\section{References}

[1] F. Arzarello, F. Olivero, D. Paola and O. Robutti, A cognitive analysis of dragging practises in Cabri environments, ZDM 34(3) (2002).

[2] I. Bartók, The envelope of Simson lines, Mathematical and Physical Journal for Secondary Schools (KÖMAL), Budapest XIV, no. 4-5 (December 1906), 93-99 (in Hungarian).

[3] T. Berta, Combination of traditional and computer based tools in mathematics education, ZDM 35(1) (2003).

[4] H. S. M. Coxeter, Introduction to Geometry, (Second printing), John Wiley \& Sons, Inc., New York - London, 1962.

[5] T. Gawlick, On Dynamic Geometry Software in the Regular Classroom, ZDM 34(3) (2002).

[6] A. P. Jahn, "Locus" and "Trace" in Cabri-géomètre: relationships between geometric and functional aspects in a study of transformation, ZDM 34(3) (2002).

[7] K. Jones, Student interpretations of a Dynamic Geometry Environment, In: European Research in Mathematics Education (Ed.: Inge Schwank), 245-258.

[8] Kadunz, G., Macros and Modules in Geometry, ZDM 34(3) (2002).

[9] J. Karsai, É. Rácz, A. Schwenk and N. Kalus, Visualization and art in the mathematics classroom, ZDM 35(1) (2003).

[10] I. László, Euklides dynamic geometry, ZDM 35(1) (2003).

[11] Mathematical and Physical Journal for Secondary Schools (KÖMAL), Budapest, Vol. 53, no. 2, February 2003, 126.

[12] F. Olivero, Cabri as a shared workspace within the proving process, Vol. 3, In: Proceedings of the 2003 Joint Meeting of PME and PMENA, Honolulu, Hawai, (N. A. Pateman, B. J. Dougherty \& J. Zilliox, eds.), 2003, 429-436.

[13] F. Olivero, Conjecturing in open geometric situations in Cabri-géomètre: an exploratory classroom experiment, Vol. 3, In: Research in Mathematics Education, London, (C. Morgan \& K. Jones, eds.), 2001, 229-246.

[14] B. Pelle, Geometry, Tankönyvkiadó, Budapest, 1979 (in Hungarian).

[15] G. Polya, Mathematical Discovery On understanding, learning, and teaching problem solving, Volume 1, John Wiley \& Sons, Inc., USA, 1966.

[16] I. Reiman, Geometry and its frontiers, Gondolat, Budapest, 1986 (in Hungarian).

[17] H. Schumann, New Standards for the Solution of Geometric Calculation Problems by Using Computers, ZDM 97(5) (1997).

Web sites

[18] Cabri Geometry, http://www.cabri.com.

[19] Cinderella, http://www.cinderella.de.

[20] Clark Kimberling's web site, http://faculty.evansville.edu/ck6/index.html.

[21] Euklides, http://www. euklides.hu/.

[22] Geometer's Sketchpad, http://www.keypress.com/sketchpad/index.html. 


$$
\text { "arkinemet" — 2004/7/23 — 13:48 — page } 13 \text { — \#13 }
$$

[23] Mathematical web site on public education (in Hungarian), http://www.sulinet.hu.

[24] Ulrich Kortenkamp: Foundations of Dynamic Geometry, Ph.D. Thesis,

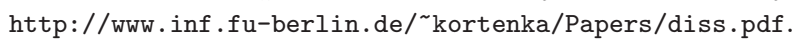

[25] Wolfram Research, http://mathworld.wolfram.com/.

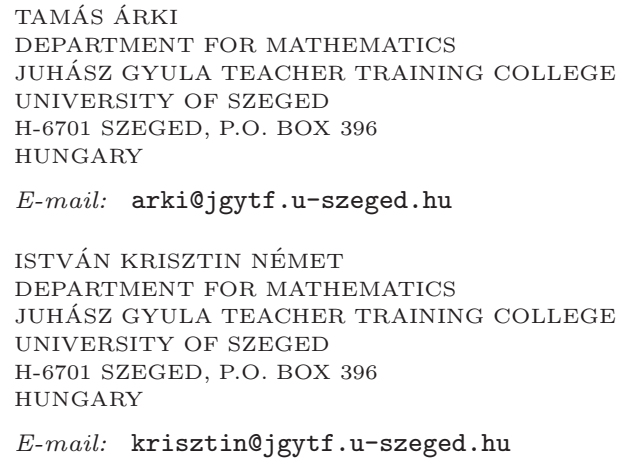

(Received July, 2003) 